“ (C) 2017 IEEE. Personal use of this material is permitted. Permission from IEEE must be obtained for all other uses, in any current or future media, including

reprinting/republishing this material for advertising or promotional purposes, creating new collective works, for resale or redistribution to servers or lists, or reuse of any copyrighted component of this work in other works." 


\title{
Time-delayed Model Predictive Direct Power Control for Vehicle to Grid and Grid to Vehicle Applications
}

\author{
Mahlagha Mahdavi Aghdam, Student Member, IEEE, Li Li, Member, IEEE, Jianguo Zhu, Senior Member, IEEE, \\ Tingting He, Student Member, IEEE, Jianwei Zhang, Student Member, IEEE \\ Centre of Green Energy and Vehicle Innovation, University of Technology Sydney, Ultimo, NSW 2007, Australia \\ Mahlagha.MahdaviAghdam@student.uts.edu.au
}

\begin{abstract}
This paper presents a time-delayed model predictive control for power converters used in vehicle to grid and grid to vehicle systems. Finite-based model predictive control has proven to be an alternate digital control method for power converters. However, there are some real-time implementation issues, including specifically time delay, that have to be addressed in order to achieve the system reliability and stability as well as better performance. The proposed method compensates the delay time arising from measuring, calculating, and applying the optimal control sequence in the digital processor. In this way, the delay time is considered in the system input and optimal switching states are applied to the converter once they are available. The proposed method is studied through two benchmarks and verified numerically via MATLAB/Simulink.
\end{abstract}

Keywords-delay compensation; electric vehicle; finite-based model predictive control; power flow control; prediction horizon; vehicle to grid; grid to vehicle;

\section{INTRODUCTION}

Electric vehicle (EV) integration to the grid has revolutionized the structure of power systems from centralized and unidirectional towards decentralized and bidirectional structure, so that the upcoming power systems will perform in a more distributed, intelligent, interactive and cooperative way. Furthermore, with advances in power electronics and modern control, the concept of the vehicle to grid (V2G) and grid to vehicle (G2V) systems by using EVs or hybrid vehicles can improve the power quality to the standards [1-4].

The advancement of modern processing and devices opened a new horizon to power converter control [5]. Modern control algorithms such as predictive control [6-9], slide mode control [10, 11], and artificial-intelligence based control [12] are applied and implemented on different power electronics' applications. Particularly, model predictive control (MPC) has gained much attention due to its flexibility, easy inclusion of uncertainties and dealing with the multi-input multi-output (MIMO) systems as well as the single-input single-output (SISO) ones. However, an accurate dynamics model of the plant and issues with real-time implementation have to be taken into consideration [13-15].

Due to the limited number of switching states in power converter, finite control set MPC (FMPC) will be a suitable approach to predict the future system's behavior. Therefore, it can be a relevant replacement to conventional control approaches for power converter with pulse width modulation (PWM) procedures [16-18]. Generally, in FMPC, an optimization problem will be solved and the best control sequence will be formed for a finite prediction horizon. The exhaustive search is carried on whereas the approximations and measurements are updated, for the next sampling time [19]. The variable switching frequency is the outcome of FMPC, even though it directly chooses the switching states. Strategies have been applied to control power converters depending on the applications. For example, in grid-connected converters, direct power control (DPC) is one of the popular strategies applied to control power flow as a result of easiness, and good transient performance [20].

While FMPC carries some benefits, the algorithm needs to be reformed for various purposes, mostly due to the variety of the plant characteristics that causes some challenges for the design. In FMPC, the main problem associated with real-time implementation (RTI) via digital signal processor (DSP) is the computational load which leads to a significant delay time. As a result, the system performance will be impaired if the delay time is not reflected in the plant model [21]. A common approach to compensate this delay is to consider the computation time and apply the chosen control sequence after the next sampling time [22]. In fact, the delay time varies in RTI for different applications. Therefore, this paper presents a new time-delayed model in which the updated control sequence will be applied to the system as soon as it becomes accessible. As a result, the performance of the controller will be improved, which enhances the stability as well as the power quality.

This study proposes a time-delayed model for FMPC algorithms which has addressed the computational delay involved with the RTI in the power converter control for microgrid applications. The conventional and time-delayed FMPC principles are discussed in Section II. The performance of the time-delayed FMPC is discussed through some study cases in Section III. Section IV is devoted to a discussion and comparison with conventional approach. Section $\mathrm{V}$ is dedicated to the conclusion and future work. 


\section{THEORY OF FMPC}

\section{A. Conventional FMPC principles}

Aforementioned, in FMPC, developing a precise dynamic model is an essential. The dynamic model of the system in discrete-time domain for conventional FMPC can be written in the state space form as

$$
\begin{gathered}
\boldsymbol{x}_{\boldsymbol{i}}(k+1)=A \boldsymbol{x}(k)+B \boldsymbol{u}_{\boldsymbol{i}}(k) \\
\boldsymbol{y}(k)=C \boldsymbol{x}(k)
\end{gathered}
$$

where $\boldsymbol{x}(k), \boldsymbol{u}(k)$, and $\boldsymbol{y}(k)$ are the state, input, and output vector at $k T_{s}$, respectively, $T_{s}$ is the sampling time, and $i$ is the number of possibilities. $\mathrm{A}, \mathrm{B}$ and $\mathrm{C}$ are discrete matrices which are derived from discretizing the continuous-time system model. The state variable $\boldsymbol{x}(k)$, is accessible via measurement. The future state variables at the step $k+N_{p}$, where $N_{p}$ is the prediction horizon, can be represented as

$$
\begin{gathered}
\boldsymbol{Y}_{\boldsymbol{i}}(\boldsymbol{k})=\left(\begin{array}{c}
C A \\
C A^{2} \\
\vdots \\
C A^{N_{p}}
\end{array}\right) \boldsymbol{x}(k) \\
+\left(\begin{array}{cccc}
C B & 0 & \ldots & 0 \\
C A B & C B & \ldots & 0 \\
\vdots & \vdots & \vdots & \vdots \\
C A^{N_{p}-1} B & \ldots & \ldots & C B
\end{array}\right) \boldsymbol{U}_{\boldsymbol{i}}(k) \\
\boldsymbol{Y}_{\boldsymbol{i}}(k)=\left[\begin{array}{lllll}
\boldsymbol{y}_{\boldsymbol{i}}(k+1) & \boldsymbol{y}_{\boldsymbol{i}}(k+2) & \ldots & \boldsymbol{y}_{\boldsymbol{i}}\left(k+N_{p}\right)
\end{array}\right]^{T} \\
\boldsymbol{U}_{\boldsymbol{i}}(k)=\left[\begin{array}{llll}
\boldsymbol{u}_{\boldsymbol{i}}(k) & \boldsymbol{u}_{\boldsymbol{i}}(k+1) & \ldots & \boldsymbol{u}_{\boldsymbol{i}}\left(k+N_{p}-1\right)
\end{array}\right]^{T}
\end{gathered}
$$

where $\boldsymbol{Y}$ and $\boldsymbol{U}$ are the output and input sequences for $N_{p}$ prediction horizon. The control objective, in the case of tracking the reference, $\boldsymbol{Y}^{*}$, can be defined as

$$
J_{i}(k)=\left\|\boldsymbol{Y}^{*}(k)-\boldsymbol{Y}_{\boldsymbol{i}}(k)\right\|_{2}^{2}
$$

The optimization problem can be solved via minimization of the objective function

$$
\boldsymbol{U}_{\text {opt }}(k)=\frac{\arg \min J_{i}(k)}{\boldsymbol{U}(k)}
$$

Succeeding to the receding horizon control (RHC) concept, in Fig.1, the first element of the optimizing sequence $\boldsymbol{U}_{\text {opt }}(k)$ is applied to the IGBT switches.

In RTI, a major part of sampling time may be used to determine the control sequence, causing a considerable delay. As the number of the possible switching states increase, the delay time will increase as well. The effect of such a delay will be reflected on the prediction, particularly when a onestep horizon algorithm is in place.

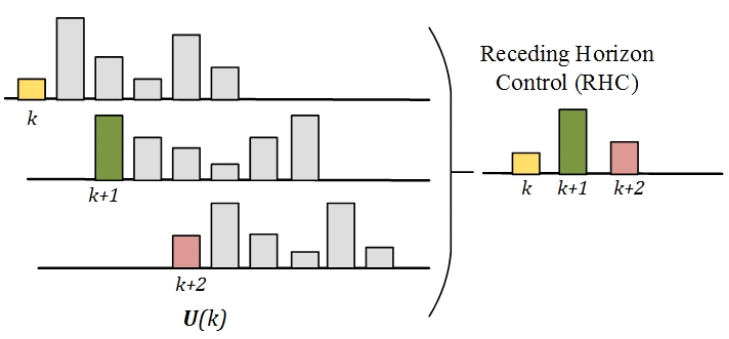

Fig.1. Receding Horizon Control Principle [23]

\section{B. Proposed Time-delayed FMPC}

Aforementioned, a general method to deal with the delay is two-step prediction ahead rather than one-step. As the delay is not constant for RTI, a time-delayed model is proposed in this paper. Based on this model, the system performance will be improved with enhanced system stability and reliability.

If we consider a delay time $\tau$ in the system input, the state space system model in continuous-time domain will be as follows,

$$
\begin{gathered}
\frac{d \boldsymbol{x}}{d t}=D \boldsymbol{x}(t)+F \boldsymbol{u}(t-\tau) \\
\boldsymbol{y}(\boldsymbol{t})=G \boldsymbol{x}(\boldsymbol{t})
\end{gathered}
$$

where $D, F$ and $G$ are matrices derived from the system model. Through discretization as can be seen in Fig. 2, by applying the delay time $\tau$ between $K$ and $K+1$, the state space model of the system can be obtained as

$$
\begin{gathered}
\boldsymbol{x}(k+1)=A \boldsymbol{x}(k)+B_{1} \boldsymbol{u}(k-1)+B_{2} \boldsymbol{u}(k) \\
\boldsymbol{y}(k)=C \boldsymbol{x}(k)
\end{gathered}
$$

Where $A, B_{1}$, and $B_{2}$ can be calculated as follow,

$$
\begin{gathered}
A=e^{D T_{S}} \\
B_{1}=e^{D\left(T_{s}-\tau\right)} \cdot \int_{0}^{\tau} e^{D \delta} \cdot F d \delta \\
B_{2}=\int_{0}^{T_{S}-\tau} e^{D \delta} \cdot F d \delta
\end{gathered}
$$

Following the RHC principle and the assumption of $\boldsymbol{u}(k)=\cdots=\boldsymbol{u}\left(k+N_{p}-1\right)$, the future state variables at the step $k+N_{p}$ can be represented as

$$
\begin{aligned}
& \boldsymbol{Y}(\boldsymbol{k}) \\
& =\left(\begin{array}{c}
C A \\
C A^{2} \\
\vdots \\
C A^{N_{p}}
\end{array}\right) \boldsymbol{x}(k)+\left(\begin{array}{c}
C B_{1} \\
C A B_{1} \\
\vdots \\
C A^{N_{p}-1} B_{1}
\end{array}\right) \boldsymbol{u}(k-1) \\
& +\left(\begin{array}{c}
C B_{2} \\
C\left(A B_{2}+B_{1}+B_{2}\right) \\
\vdots \\
\sum_{l=0}^{N_{p}-2}
\end{array}\right) \boldsymbol{u}(k) \\
& \boldsymbol{Y}(\boldsymbol{k})=\left[\begin{array}{ll}
\left.N^{N_{p}-l-1}+A^{N_{p}-l-2} B_{1}\right)+C B_{2}
\end{array}\right) \\
&
\end{aligned}
$$




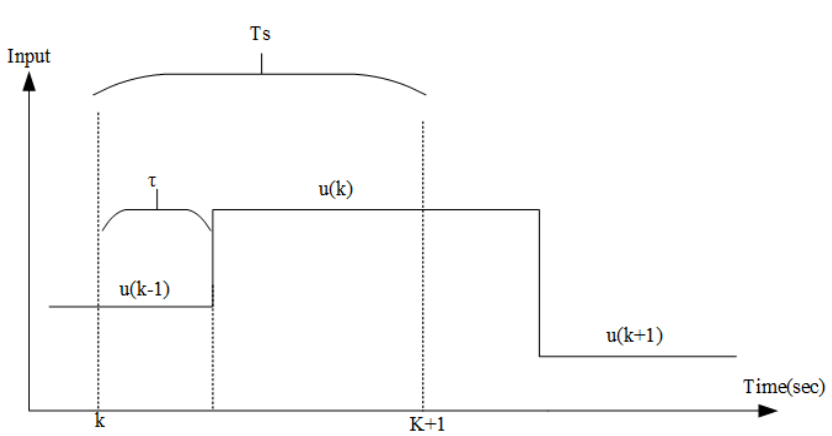

Fig.2. Graphical Representation of Time-delayed MPC

\section{CASE STUDIES}

As we know, for $r$ level- $s$ phase ( $r \mathrm{~L}-s \mathrm{Ph})$ power converters, there are $m=r^{s}$ possible voltage vectors. To examine the proposed model, a $2 \mathrm{~L}-3 \mathrm{Ph}$ voltage source inverter (VSI) and a $3 \mathrm{~L}-3 \mathrm{Ph}$ neutral point clamped (NPC) VSI are chosen as benchmarks. Both inverters are assumed to be gridconnected.

\section{A. FMPC with the Conventional Approach}

\section{1) $2 L-3 P h$ Grid-connected VSI}

The structure of the $2 \mathrm{~L}-3 \mathrm{Ph}$ grid-connected inverter is represented in Fig. 3. The inverter consists of three paired IGBT switches. The inverter's output voltage vectors are described by

$$
v_{m}=\left\{\begin{aligned}
\frac{2}{3} e^{j(m-1) \frac{\pi}{3}} V_{d c}, & & m=1, \ldots, 6 \\
0, & m & =0,7
\end{aligned}\right.
$$

The mathematical model of this case can be formulated in the stationary frame via matrix $M$ (Clarke's transformation), provided in the appendix, as

$$
V_{m}^{\alpha \beta}=L \frac{d i_{o}^{\alpha \beta}}{d t}+R \cdot i_{o}^{\alpha \beta}+V_{\text {grid }}^{\alpha \beta}
$$

Furthermore, for a balanced and positive sequence system, active power $P$ and reactive power $Q$ can be obtained as

$$
\begin{aligned}
& P=\frac{3}{2}\left(V_{\text {grid }}^{\alpha} i_{0}^{\alpha}+V_{\text {grid }}^{\beta} i_{0}^{\beta}\right) \\
& Q=\frac{3}{2}\left({V_{\text {grid }}}^{\beta} i_{0}^{\alpha}-V_{\text {grid }}^{\alpha} i_{0}^{\beta}\right)
\end{aligned}
$$

By taking the first derivative from (10) the system model can be achieved based on active and reactive power. The system model is in the non-linear form, therefore, to predict the future behavior of the system, linearization approach is applied. The matrices $D$ and $F$ are provided in the Appendix while the state variable and input are defined as follows,

$$
\begin{gathered}
\boldsymbol{x}=\left[\begin{array}{lll}
P & Q & V_{\text {grid }}{ }^{\alpha} V_{\text {grid }}^{\beta}
\end{array}\right]^{T} \\
\boldsymbol{u}=\left[\begin{array}{lll}
V_{m}{ }^{\alpha} & V_{m}^{\beta}
\end{array}\right]^{T}
\end{gathered}
$$

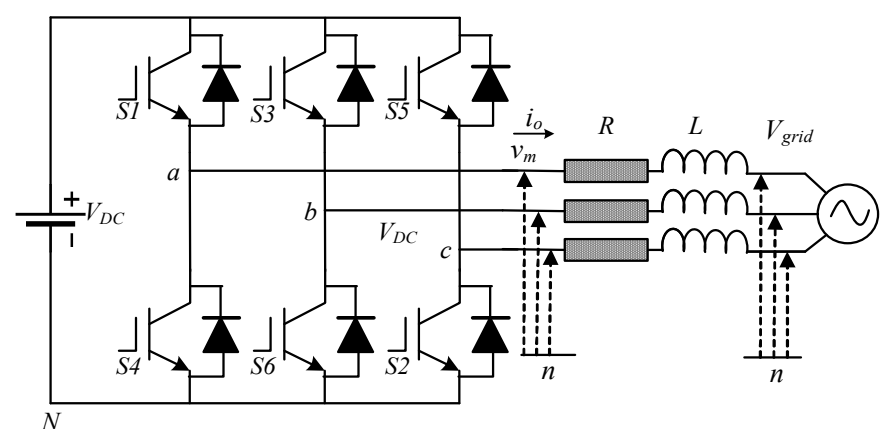

Fig.3. 2L-3Ph Grid-connected VSI

The objective function has two terms including power flow control and switching frequency reduction as follows

$$
\begin{gathered}
J=\operatorname{Err}_{P w r}(k+2)+\lambda S_{E r r} \\
\operatorname{Err}_{P w r}(k+2)=\left[P^{*}(k+2)-P(k+2)\right]^{2} \\
+\left[Q^{*}(k+2)-Q(k+2)\right]^{2} \\
S_{E r r}=\sum_{h=a, b, c}\left(\begin{array}{l}
\left(S_{h}(k+2)-S_{h}(k+1)\right)^{2}
\end{array}\right.
\end{gathered}
$$

where $\operatorname{Err}_{P w r}$ is the error between the reference and predicted power, and $S_{E r r}$ is the switching times between the adjacent switching states. As can be seen, two-step prediction is utilized for delay compensation.

\section{2) 3L-3Ph NPC Grid-connected VSI}

The topology of the $3 \mathrm{~L}-3 \mathrm{Ph}$ NPC grid-connected inverter is represented in Fig. 4. The inverter consists of six complimentary pairs of IGBT switches. Therefore, there are 27 possibilities, including three zero, twelve small, six medium and six large voltage vectors as illustrated in Fig.5. The output voltage of the inverter in stationary frame are described by

$$
\begin{aligned}
& V_{\alpha \beta(N P C) \xi}=\frac{V_{D C}}{2} M S_{\xi} \\
& S_{\xi}=\left[\begin{array}{lll}
S_{\xi a} & S_{b \xi} & S_{c \xi}
\end{array}\right]^{T}
\end{aligned}
$$

where $\mathrm{M}$ is the Clarke's transformation matrix, $V_{D C}$ is the DClink voltage and $S_{\xi}$ is the switching signal for $\xi=0, \ldots, 26$.

Although the mathematical model of this inverter for power flow control is same as the pervious case, balance of the neutral point is a compulsory action for achieving the high quality power. There are two capacitors in this topology which serve as a voltage divider of full DC-Link $\left(V_{D C}\right)$. The neutral current can be obtained as

$$
i_{N}=i_{C U}-i_{C L}=C_{U} \frac{d V c_{U}}{d t}-C_{L} \frac{d V c_{L}}{d t}
$$

where $C_{U}$ and $C_{L}$ are the capacitance of the upper and lower capacitors, respectively. Assume $C_{U}=C_{L}=C$ and $\Delta V_{C}=$ $V_{C U}-V_{C L}$ then,

$$
i_{N}=C \frac{d\left(V c_{U}-V c_{L}\right)}{d t}=C \frac{d \Delta V_{c}}{d t}
$$




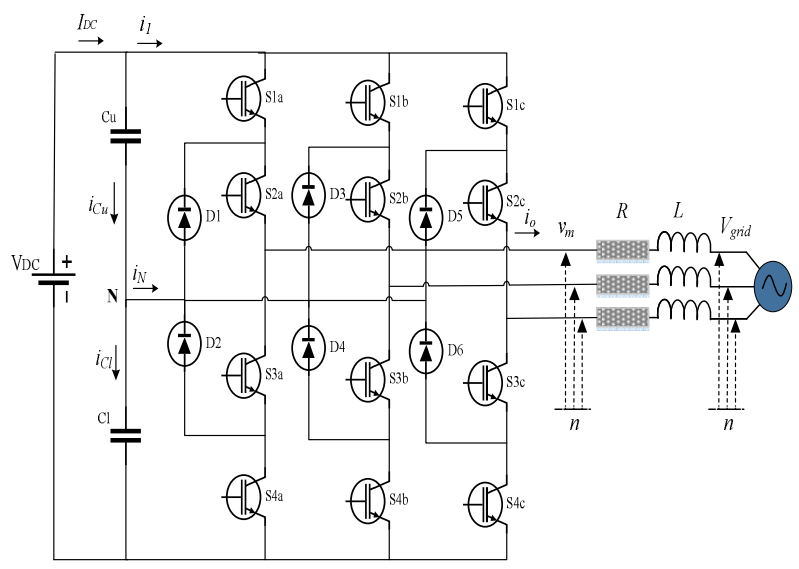

Fig.4. 3L-3Ph NPC Grid-connected VSI

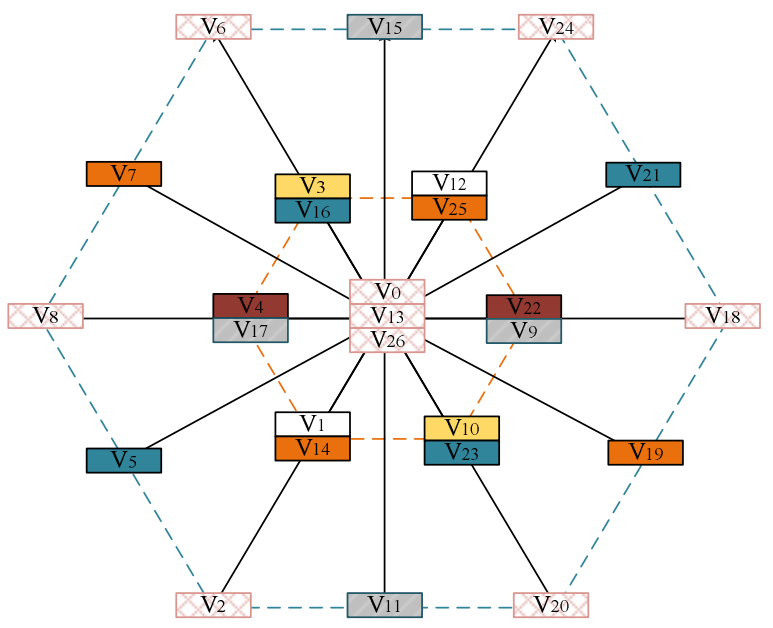

Fig.5. Voltage Vectors of 3L-3Ph NPC Grid-connected VSI

After discretizing and shifting one step forward, the future value of balanced voltage leads to

$$
\Delta V_{c}(k+2)=\frac{T_{s}}{C} i_{N}(k+2)+\Delta V_{c}(k+1)
$$

As a result, for power flow control and switching frequency reduction the cost function will have three terms including (17) as follow

$$
J=\operatorname{Err}_{P w r}(k+2)+\lambda_{1} S_{E r r}+\lambda_{2} \Delta V_{c}(k+2)
$$

\section{B. Proposed Time-delayed FMPC}

In DSP-based RTI, $\tau$ can be characterized as

$$
\begin{gathered}
\tau=\tau_{\text {constant }}+\tau_{\text {comp }} \\
\tau_{\text {constant }}=\tau_{\text {mes }}+\tau_{\text {apply }}
\end{gathered}
$$

where $\tau_{\text {mes }}, \tau_{\text {comp }}$ and $\tau_{\text {apply }}$ are the time needed for measurements and analog-to-digital conversion, calculation and application of the control sequence, respectively. Here, $\tau_{\text {mes }}$ and $\tau_{\text {apply }}$ can be assumed constant and reliant on the DSP device, while $\tau_{\text {comp }}$ has the major effect on the performance of the system.
The flowchart of time-delayed FMPC is depicted in Fig.6. After grid voltage and line current measurements and power calculation, the resulting computational time $\tau_{\text {comp }}$ is added to the constant delay time $\tau_{\text {constant }}$, which gives the total delay time for RTI based on individual application. By comparing $T_{S}$ and $\tau$ the number of prediction horizon is obtained.

Next, based on the calculated $\tau$, the discrete-time domain $A, B_{1}$, and $B_{2}$ matrices are calculated. Then the optimization problem is solved based on exhaustive search for $m$ possibilities $\left(m=r^{s}\right)$ to attain the optimal control sequence.

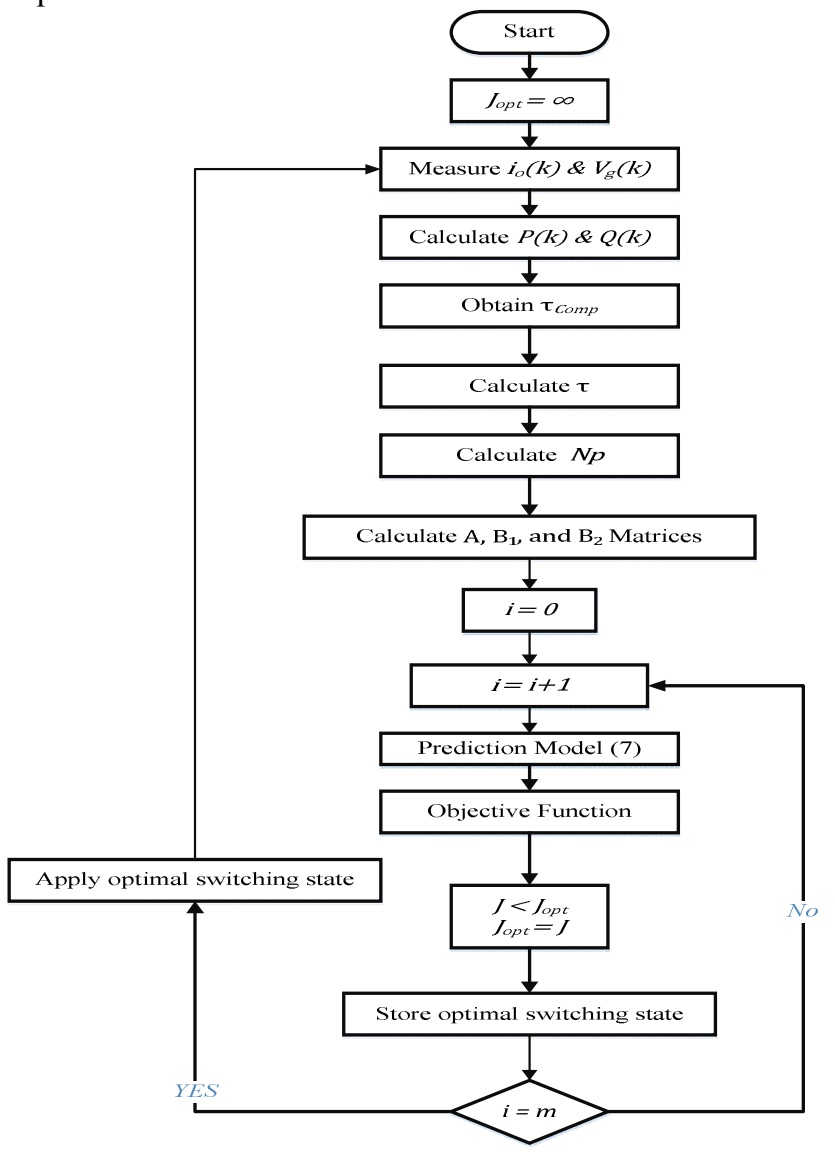

Fig.6. Flowchart of Time-delayed FMPC

\section{RESULTS, COMPARISON AND DISCUSSION}

\section{A. Simulation Results}

Simulation of a $2 \mathrm{~L}-3 \mathrm{Ph}$ and a $3 \mathrm{~L}-3 \mathrm{Ph}$ NPC girdconnected inverter with RL filters and loads is executed by using MATLAB/Simulink. The system parameters listed in Table I.

The initial active and reactive power is set to zero. The active power reference is increased from 0.5 to $2 \mathrm{~kW}$ at $0.01 \mathrm{~s}$ and decreased from 2 to $-1 \mathrm{~kW}$ at $0.02 \mathrm{~s}$. After that, the active power reference is kept to $-1 \mathrm{~kW}$ till $0.03 \mathrm{~s}$ and then is kept at $0.5 \mathrm{~kW}$. The reactive power reference is altered to -1 , 0.5 , and $1 \mathrm{kVAr}$ at $0.04 \mathrm{~s}, 0.05 \mathrm{~s}$ and $0.06 \mathrm{~s}$, respectively. 
Fig. 7 demonstrates the controller performances for a 2L-3Ph VSI with conventional and time-delayed FMPC. Furthermore, Fig. 8 shows the active and reactive power flow for a $3 \mathrm{~L}-3 \mathrm{Ph}$ NPC-VSI with conventional and time-delayed FMPC.
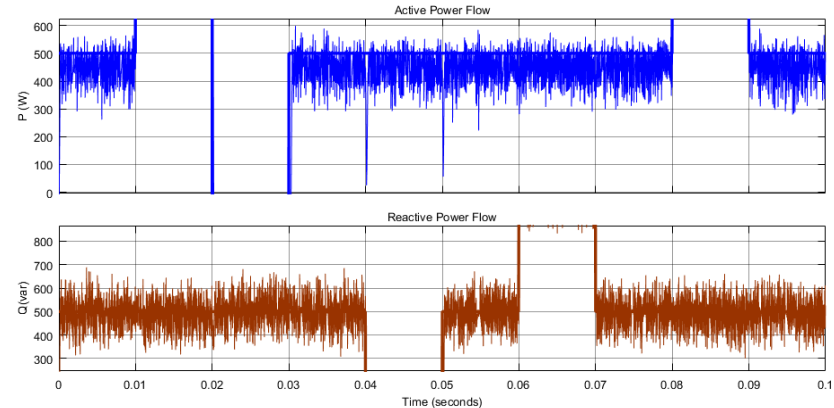

(a)
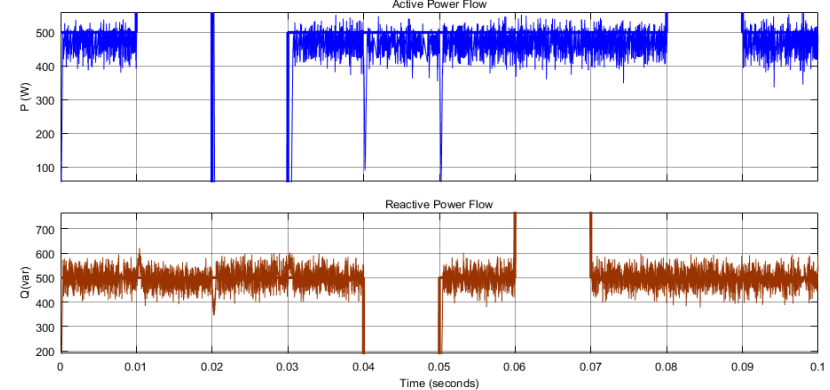

(b)

Fig.7. Numerical simulation results for 2L-3Ph VSI a) Power Ripples of FMPC with two-step prediction horizon b) Power Ripples of Time-delayed FMPC $(\tau=27 \mu \mathrm{sec})$
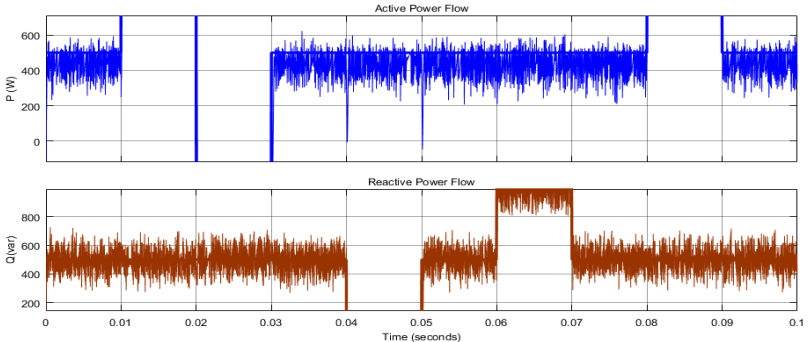

(a)
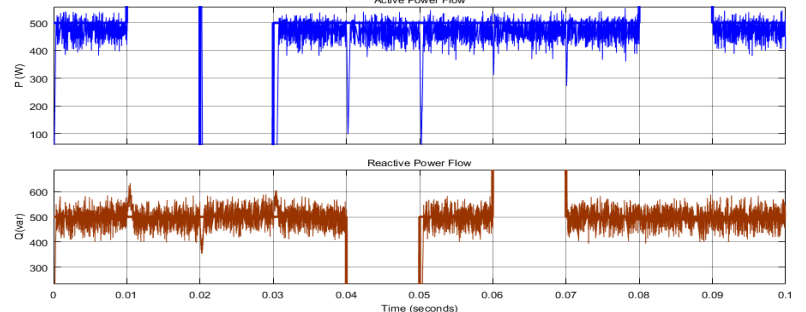

(b)

Fig.8. Numerical simulation results for 3L-3Ph NPC VSI a) Power Ripples of FMPC with two-step prediction horizon b) Power Ripples of Time-delayed $\operatorname{FMPC}(\tau=27 \mu \mathrm{sec})$

As can be seen, the power flow tracking shows the better transient and steady state performance for the proposed model than the conventional one. In order to get a better evaluation of the proposed control algorithm, quantitative comparison has been conducted.

\section{B. Quantitative Comparison and Discussion}

A quantitative comparison of the conventional FMPC approach and time-delayed FMPC with $\tau_{\text {comp }}=27 \mu$ sec is carried out for both case studies. With the intention of conducting a better comparison of the control approaches, indices like the average switching frequency, and power ripples, overshoot, settling and rise time are tabulated in Table II. The quantities are measured through MATLAB.

For clearer quantitative comparison, the indices are normalized. $P_{\text {avrg }}$ and $Q_{\text {avrg }}$ are the average active and reactive power which are considered $1 \mathrm{~kW}$ and $1 \mathrm{kVar}$, correspondingly. $P_{\text {rip }}$, is obtained from the active power ripple divided by average active power. $Q_{\text {rip }}$, is obtained from the reactive power ripple divided by average reactive power. In the same manner, settling time is normalized by the value of $T_{s}$.

It can be realized that the by applying the timedelayed compensation method, the power ripples have been reduced by $41.19 \%$. The power factor can be achieved as close as unity by both methods, but FMPC with time-delayed approach is able to track the reference more precisely, with less power ripples. However, the switching loss has increased slightly, by only $4.4 \%$.

Additionally, the proposed model has shown a good performance to different value of time which is considered for delay. The results are listed in Table III. Moreover, the performance indices show that the controller is reliable and works effectively.

\begin{tabular}{|c|c|c|}
\hline Filter resistance & $R$ & $1 \Omega$ \\
\hline Filter inductance & $L$ & $10 \mathrm{mH}$ \\
\hline Grid voltage & $V_{\text {grid }}$ & $120 \mathrm{~V}$ \\
\hline Voltage frequency & $f$ & $50 \mathrm{~Hz}$ \\
\hline Sampling period & $T_{S}$ & $25 \mu s$ \\
\hline Constant delay & $\tau_{\text {constant }}$ & $7 \mu s$ \\
\hline DC source voltage (1) & $V_{D C 1}$ & $400 \mathrm{~V}$ \\
\hline DC source voltage (2) & $V_{D C 2}$ & $700 \mathrm{~V}$ \\
\hline
\end{tabular}

\begin{tabular}{|c|c|c|c|c|}
\multicolumn{4}{c}{ TABLE II. Quantitative Comparison } \\
\hline \multirow{2}{*}{ Indices } & \multicolumn{2}{|c|}{ FMPC } & \multicolumn{2}{c|}{ Time-delayed FMPC } \\
\cline { 2 - 5 } & $\begin{array}{c}2 \mathrm{~L}-3 \mathrm{Ph} \\
\text { VSI }\end{array}$ & $\begin{array}{c}3 \mathrm{~L}-3 \mathrm{Ph} \\
\text { NPC VSI }\end{array}$ & $\begin{array}{c}2 \mathrm{~L}-3 \mathrm{Ph} \\
\text { VSI }\end{array}$ & $\begin{array}{c}3 \mathrm{~L}-3 \mathrm{Ph} \\
\text { NPC VSI }\end{array}$ \\
\hline $\boldsymbol{f}_{\boldsymbol{s}}(\mathrm{Hz})$ & $40 \mathrm{k}$ & $40 \mathrm{k}$ & $40 \mathrm{k}$ & $40 \mathrm{k}$ \\
\hline $\boldsymbol{f}_{\text {sw }}(\mathrm{Hz})$ & $5.66 \mathrm{k}$ & $6.89 \mathrm{k}$ & $5.48 \mathrm{k}$ & $6.568 \mathrm{k}$ \\
\hline $\boldsymbol{P}_{\text {rip }}$ & 0.696 & 0.823 & 0.409 & 0.483 \\
\hline $\boldsymbol{Q}_{\text {rip }}$ & 0.591 & 0.712 & 0.347 & 0.418 \\
\hline Settling Time & 0.4004 & 0.5892 & 0.3428 & 0.546 \\
\hline Overshoot & $47.146 \%$ & $38.98 \%$ & $44.13 \%$ & $37.11 \%$ \\
\hline
\end{tabular}

TABLE III. Time-delayed FMPC with Different $\boldsymbol{\tau}$

\begin{tabular}{|c|c|c|c|c|}
\hline Indices & \multicolumn{4}{|c|}{ Time-delayed FMPC } \\
\hline \multirow[t]{2}{*}{$\tau_{\text {comp }}$} & \multicolumn{2}{|c|}{ 2L-3Ph VSI } & \multicolumn{2}{|c|}{ 3L-3Ph NPC VSI } \\
\hline & $8 \mu \mathrm{sec}$ & $38 \mu \mathrm{sec}$ & $38 \mu \mathrm{sec}$ & $48 \mu \mathrm{sec}$ \\
\hline$f_{s w}(H z)$ & $5.66 \mathrm{k}$ & $5.06 \mathrm{k}$ & $6.568 \mathrm{k}$ & $6.168 \mathrm{k}$ \\
\hline $\boldsymbol{P}_{\text {rip }}$ & 0.106 & 0.126 & 0.171 & 0.188 \\
\hline$Q_{\text {rip }}$ & 0.126 & 0.139 & 0.259 & 0.268 \\
\hline Settling Time & 0.31 & 0.406 & 0.551 & 0.559 \\
\hline Overshoot & $43.13 \%$ & $46.11 \%$ & $37.59 \%$ & $37.98 \%$ \\
\hline
\end{tabular}




\section{CONCLUSION AND FUTURE WORK}

A time-delayed FMPC is presented in this study for vehicle to grid and grid to vehicle applications where a delay is considered in the input of the system model. This approach has shown a better transient and steady state performance while reducing the power ripples significantly for power converters in distributed generation. The proposed method compensates the delay time resulting from DSPs' computation and implementation of the control signal. The delay time in this work has been estimated through trial and error, which in the future work can be included into algorithm. Another concern about the FMPC approach is the weighting factor design, which needs a deeper investigation. Nevertheless, industrial approval of MPC in power converters and drives has yet to come. In [24], the authors assessed the technology readiness of MPC with conclusion that MPC will perform a pivotal key for the next generation of power converters and electrical drives to operate in a more reliable, stable, and efficient way.

\section{APPENDIX}

Clarke's mathematical transformation matrix, $\mathrm{M}$, is defined as follow,

$$
M=\frac{2}{3}\left[\begin{array}{rrr}
1 & -\frac{1}{2} & -\frac{1}{2} \\
0 & \frac{\sqrt{3}}{2} & -\frac{\sqrt{3}}{2}
\end{array}\right]
$$

$D$ and $F$ are the space state matrixes in continuous-time domain.

$$
\begin{gathered}
D=\left(\begin{array}{cccc}
\frac{-R}{L} & -\omega & \frac{-6 \bar{V}_{\text {gird }}^{\alpha}}{2 L}+\frac{3 \bar{V}_{m}^{\alpha}}{2 L} & \frac{-6 \bar{V}_{\text {gird }}^{\beta}}{2 L}+\frac{3 \bar{V}_{m}^{\beta}}{2 L} \\
\omega & \frac{-R}{L} & -\frac{3 \bar{V}_{m}^{\beta}}{2 L} & \frac{3 \bar{V}_{m}^{\alpha}}{2 L} \\
0 & 0 & 0 & -\omega \\
0 & 0 & 0
\end{array}\right) \\
F=\frac{3}{2 L}\left(\begin{array}{cc}
\bar{V}_{\text {gird }}^{\alpha} & \bar{V}_{\text {gird }}^{\beta} \\
\bar{V}_{\text {gird }}^{\beta} & -\bar{V}_{\text {gird }}^{\alpha} \\
0 & 0 \\
0 & 0
\end{array}\right)
\end{gathered}
$$

where $\bar{z}$ denotes the currently available value of $z(),. z=$ $V_{\text {gird }}^{\alpha}, V_{\text {gird }}^{\beta}, V_{m}^{\alpha}, V_{m}^{\beta}$.

\section{REFERENCES}

[1] O. Palizban and K. Kauhaniemi, "Hierarchical control structure in microgrids with distributed generation: Island and grid-connected mode," Renewable and Sustainable Energy Reviews, vol. 44, pp. 797-813, 2015.

[2] C. Buccella, C. Cecati, and H. Latafat, "Digital control of power converters-A survey," IEEE Transactions on Industrial Informatics, vol. 8, pp. 437-447, 2012.

[3] L. Mariam, M. Basu, and M. F. Conlon, "Microgrid: Architecture, policy and future trends," Renewable and Sustainable Energy Reviews, vol. 64, pp. 477-489, 2016.

[4] U. K. Madawala and D. J. Thrimawithana, "A bidirectional inductive power interface for electric vehicles in V2G systems," IEEE Transactions on Industrial Electronics, vol. 58, pp. 47894796, 2011.
[5] S. Kouro, M. A. Perez, J. Rodriguez, A. M. Llor, and H. A. Young, "Model Predictive Control: MPC's Role in the Evolution of Power Electronics," IEEE Industrial Electronics Magazine, vol. 9, pp. 821, 2015.

6] Rivera, J. Rodriguez, and S. Vazquez, "Predictive control in power converters and electrical drives - part ii [guest editorial]," IEEE Transactions on Industrial Electronics, vol. 63, pp. 44724474, 2016.

7] M. Rivera, J. Rodriguez, and S. Vazquez, "Predictive Control in Power Converters and Electrical Drives-Part I," IEEE Transactions on Industrial Electronics, vol. 63, pp. 3834-3836, 2016.

8] M. Rivera, J. Rodriguez, and S. Vazquez, "Predictive Control in Power Converters and Electrical Drives-Part IV," IEEE Transactions on Industrial Electronics, vol. 63, pp. 5804-5806, 2016.

[9] M. Rivera, V. Yaramasu, J. Rodriguez, and B. Wu, "Model predictive current control of two-level four-leg inverters-Part II: Experimental implementation and validation," IEEE Transactions on Power Electronics, vol. 28, pp. 3469-3478, 2013.

[10] R. Guzman, L. G. de Vicuna, J. Morales, M. Castilla, J. Miret, and J. Torres-Martinez, "Sliding-mode control for a three-phase shunt active power filter in natural frame," in Industrial Electronics Society, IECON 2015-41st Annual Conference of the IEEE, 2015, pp. 001211-001216.

11] J. J. More, P. F. Puleston, C. Kunusch, and M. A. Fantova, "Development and implementation of a supervisor strategy and sliding mode control setup for fuel-cell-based hybrid generation systems," Energy Conversion, IEEE Transactions on, vol. 30, pp. 218-225, 2015. C. Bhattacharjee and B. K. Roy, "Advanced fuzzy power extraction control of wind energy conversion system for power quality improvement in a grid tied hybrid generation system," IET Generation, Transmission \& Distribution, vol. 10, pp. 1179-1189, 2016.

C. Bordons and C. Montero, "Basic Principles of MPC for Power Converters: Bridging the Gap Between Theory and Practice," IEEE Industrial Electronics Magazine, vol. 9, pp. 31-43, 2015.

S. Di Cairano, "An industry perspective on MPC in large volumes applications: Potential Benefits and Open Challenges," IFAC Proceedings Volumes, vol. 45, pp. 52-59, 2012.

J. Rodriguez, M. P. Kazmierkowski, J. R. Espinoza, P. Zanchetta, H. Abu-Rub, H. A. Young, et al., "State of the art of finite control set model predictive control in power electronics," IEEE Transactions on Industrial Informatics, vol. 9, pp. 1003-1016, 2013.

M. S. Dahidah, G. Konstantinou, and V. G. Agelidis, "A review of multilevel selective harmonic elimination PWM: formulations, solving algorithms, implementation and applications," IEEE Transactions on Power Electronics, vol. 30, pp. 4091-4106, 2015.

T. Geyer, "A comparison of control and modulation schemes for medium-voltage drives: Emerging predictive control concepts versus PWM-based schemes," IEEE Transactions on Industry Applications, vol. 47, pp. 1380-1389, 2011.

J. Holtz, "Advanced PWM and Predictive Control-An Overview," IEEE Transactions on Industrial Electronics, vol. 63, pp. 3837-3844, 2016.

T. Geyer and D. E. Quevedo, "Performance of multistep finite control set model predictive control for power electronics," Power Electronics, IEEE Transactions on, vol. 30, pp. 1633-1644, 2015.

[20] J. Hu, J. Zhu, and D. G. Dorrell, "In-depth study of direct power control strategies for power converters," Power Electronics, IET, vol. 7, pp. 1810-1820, 2014.

[21] P. Karamanakos, T. Geyer, and R. Kennel, "A Computationally Efficient Model Predictive Control Strategy for Linear Systems With Integer Inputs," IEEE TRANSACTIONS ON CONTROL SYSTEMS TECHNOLOGY, 2015

[22] P. Cortes, J. Rodriguez, C. Silva, and A. Flores, "Delay compensation in model predictive current control of a three-phase inverter," Industrial Electronics, IEEE Transactions on, vol. 59, pp. 1323-1325, 2012.

[23] M. Mahdavi Aghdam, L. Li, and J. Zhu, "A Model Predictive Power Control Method with Longer Prediction Horizon for Distributed Power Generations " in Control, Automation, Robotics and Vision (ICARCV), 2016 14th IEEE Conference on, Phuket, Thailand, 2016.

[24] G. Papafotiou, G. Demetriades, and V. Agelidis, "Technology Readiness Assessment of Model Predictive Control in Medium-and High-Voltage Power Electronics," IEEE Transactions on Industrial Electronics, vol. 63, pp. 5805-5815, 2016. 